Original Paper

\title{
Axial Wall Slits Effect on the Helical Flow in the Gap between two Concentric Cylinders
}

\author{
Liu Dong ${ }^{1}$, Yang Xiao-yong ${ }^{1}$, Ding Jian ${ }^{1}$, and Kim Hyoung-Bum ${ }^{2}$ \\ ${ }^{1}$ School of Energy and Power Engineering, Jiangsu University \\ Zhenjiang 212013, China,liudong@ujs.edu.cn \\ ${ }^{2}$ School of Mechanical and Aerospace Engineering Gyeongsang National University, \\ Jinju, Gyeongnam, 660-701, South Korea, kimhb@gnu.ac.kr
}

\begin{abstract}
The helical flow regime was investigated by using DPIV when the rotating Reynolds number is small. The wall slits were azimuthally located along the inner wall of outer cylinder and the slits number of each model was 9 and 18 , another plain wall model was also studied for comparison purpose. The helical vortex flow regime can be observed in all the three models. The negative temperature gradients determine the direction of the rotation and movement of the helical vortex. But the helical wavy vortex flow can only be found in the plane and 9-slit models. And the result showed that the existence of slit wall accelerated the transition process.
\end{abstract}

Keywords: Helical flow regime; Slit wall; DPIV; Negative temperature gradient; Flow transition

\section{Introduction}

The rotating flow in the annular gap between two concentric cylinders exhibits interesting flow instability phenomena when the difference of the angular velocities of the inner cylinder and outer cylinder increases above a certain critical Reynolds number. This flow is called a Taylor-Couette flow after the pioneering work by Taylor [1]. Since then, many researchers have studied the instability causing Taylor vortices. Different methods were given for solving this eigenvalue problem by Jones [2], Wereley and Lueptow [3], Rigopoulos and Sheridan et al. [4], Marques and Lopez [5]. These studies have been performed under plain wall and isothermal conditions. A few works have been addressed on the flow transition process under non-isothermal conditions. Especially little experimental work was done.

Lee et al. [6] studied Taylor-Couette system with different numbers of slits in the outer cylinder by using DPIV method. However, their study was limited to the isothermal conditions. The temperature of the two rotating cylinders need not remain the same in many engineering problems. Lepiller et al. [7] studied the influence of radial temperature heating on the stability of the circular Couette flow, in their experiment, the Grashof number varied from -1000 to 1000 , and they found that a radial temperature gradient destabilizes the Couette flow leading to a pattern of traveling helicoidally vortices occurring only near the bottom of the system. They also found that the size of the pattern increases as the rotation frequency of the cylinder is increased. Hayase et al. [8] studied the flow and heat transfer in the space between two coaxial cylinders by using numerical calculation. Lee and Minkowycz [9] studied heat transfer characteristics by using the naphthalene sublimation technique in the annular gap between two short concentric cylinders which had either two smooth walls or one smooth and one axially slit wall. This study yielded qualitative information regarding heat transfer but did not address the flow phenomena inside the annular gap. Liu et al [11] studied the different temperature gradient effect on the flow transition process, and found the buoyant force due to the temperature gradient clearly generated the helical flow when the rotating Reynolds number is small

Although many works focus on the Taylor-Couette flow and some of them consider the temperature gradient and wall shape effect, there is little work on the wall shape effects on helical flow regime. The present work explored the presence of slits on the transition process of the helical flow. Digital particle image velocimetry (DPIV) was used to measure the flow field inside the gap. We believe this study can help understand flow instability phenomena in a Taylor-Couette flow.

\section{Apparatus and experimental method}

The geometries of the experimental models used in this study are shown in Fig.1. The inner cylinder was made of aluminum

Received April 25 2012; Accepted for publication April 17 2012: Paper number O12007S

Corresponding author: Liu Dong, Associate Professor, liudong@ujs.edu.cn

This paper was presented at 11th Asian International Conference on Fluid Machinery (AICFM) and $3^{\text {rd }}$ Fluid Power Technology Exhibition, November 21-23, 2011, Chennai, India 
pipe with black coating to prevent the reflection of a laser, which has an external radius $r_{i}=33 m m$. The outer cylinder was made of acryl and had an inner radius $r_{0}=40 \mathrm{~mm}$. Three models were studied in this paper, one model was a plain model with no slit in the wall, the other models were wall slits model, which have 9 and 18 slits in the outer cylinder. The annular gap (d) between the cylinders was $7 \mathrm{~mm}$ and the length of the cylinders $(\mathrm{L})$ was $336 \mathrm{~mm}$. The radius ratio $\left(\mathrm{r}_{\mathrm{i}} / \mathrm{r}_{\mathrm{o}}\right)$ and aspect ratio (L/d) were 0.825 and 48, respectively, following the studies of Cole [10] and Wereley and Lueptow [3]. The depth of each slit was 5mm. The inner cylinder was rotated with a belt and pulley driven by a micro stepping motor having a resolution of 400,000 steps per revolution. This micro stepper motor was controlled through a computer, which allowed for the precise control of angular velocity $(\Omega)$ and the acceleration to the preset velocity.

a

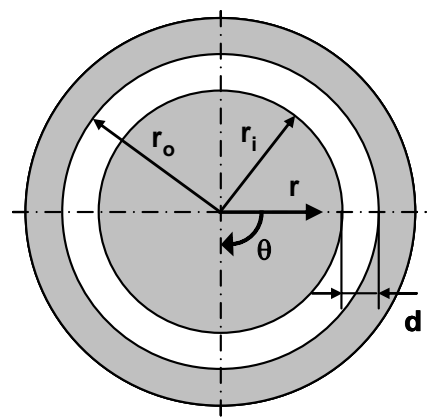

b

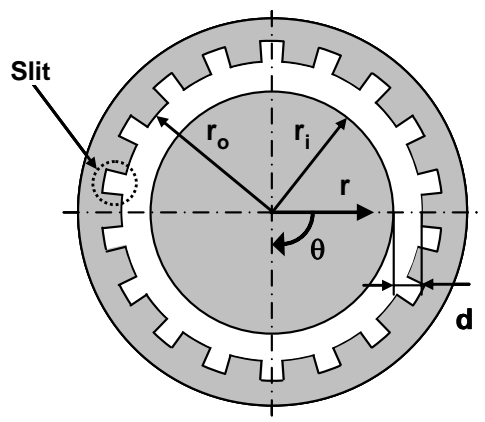

C

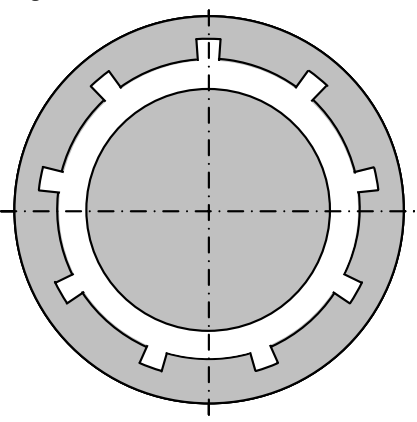

Fig. 1 Geometries of experimental models (a) Plain model (b) 18-Slit model (c) 9-Slit model

Fig.2. shows the experimental apparatus and set-up to measure the velocity field. The working fluid sodium iodide solution filled the annular gaps and the space between the outer cylinder and the enclosure box in order to avoid the light refraction effect. The details of this method were explained in a previous study by Lee et al. [6]. Two heat exchangers were installed inside the inner cylinder and in the gap between the enclosure box and the outer cylinder, which were connected with constant temperature baths and, by adjusting the temperature and the flow rate of constant temperature bath, different temperature gradient could be generated between two cylinders.

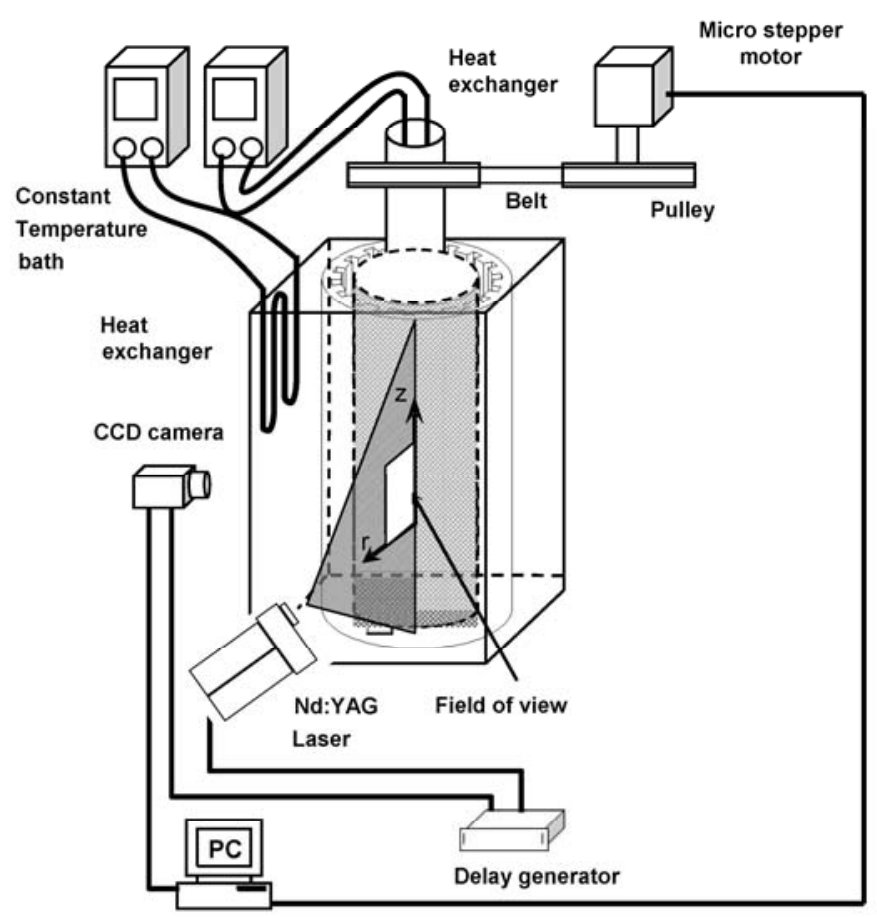

Fig.2 Schematic diagram of experimental apparatus

The effect of the temperature gradient is parameterized by the Grashof number, defined as $G r=d^{3} \beta g \Delta T / v^{2}$, where g is the acceleration due to gravity, $\beta$ is the coefficient of the thermal expansion of the working fluid, and $\Delta T$ is the temperature 
change across the gap. $\Delta T$ is defined as $\left(T_{i}-T_{o}\right)$ and is considered to be a negative temperature gradient if the inner cylinder is hotter than the outer cylinder. In this research, the temperature gradient $(\Delta T)$ was $6^{\circ} \mathrm{C}$, and the Grashof number was 6700 . In this experiment, the rotational speed was expressed as a rotating Reynolds number, which is defined as $R e=r_{i} d \Omega / v$, where $\Omega$ is the angular velocity of the inner cylinder.

\section{Experimental results and discussion}

Radial and axial instantaneous velocity fields between the inner and outer cylinders were measured using the DPIV method. The axial position, $\mathrm{Z}^{*}=\mathrm{z} / \mathrm{d}$, and the radial position, $\mathrm{R}^{*}=\left(r_{o}-r_{i}\right) / d$, were normalized by the annular gap width. Therefore, the latter $\left(\mathrm{R}^{*}\right)$ varied from 0 at the wall of the inner cylinder to 1 at the wall of outer cylinder of a plain model. The region $\mathrm{R}^{*}>1$ refers to the axial slit space in the outer cylinder. Flow previous study [11], two different helical flow regimes appeared, one is helical vortex flow ,and the other is helical wavy vortex flow, we will study these flow regimes by measuring the velocity fields while increasing the Reynolds number.

\subsection{Helical Vortex Flow Regime}

Fig.3 shows the instantaneous flow fields of this flow regime at Re=96 in different models when $\mathrm{Gr}$ is 6700 , the time interval of each velocity field is $0.4 \mathrm{~s}$. The background represents the vorticity distribution, which was calculated from the radial velocity $v_{r}$ and axial velocity $v_{z}$ using the equation $\omega=d v_{r} / d v_{z}-d v_{z} / d v_{r}$.Affected by this negative temperature gradient, the vortex pairs have the tendency to move to the bottom direction in both models. For the plain model, this flow regime first occurred at Re $=69$, but for the 9-slit and 18-slit models, which first occurred at Re=57. From this result, we can see that the existence of slit in the out cylinder wall accelerated the transition to this helical vortex flow. Comparing the radial positions of the vortex centers in the vortex pair of the plain and 18-slit models, the vortex centers of each vortex nearly always have the same radial position in the plain model, while the center of the clockwise vortex in the vortex pairs is located closer to the inner cylinder under the negative temperature gradient. No obvious vortex was found in the slit space at this flow regime.

(a)
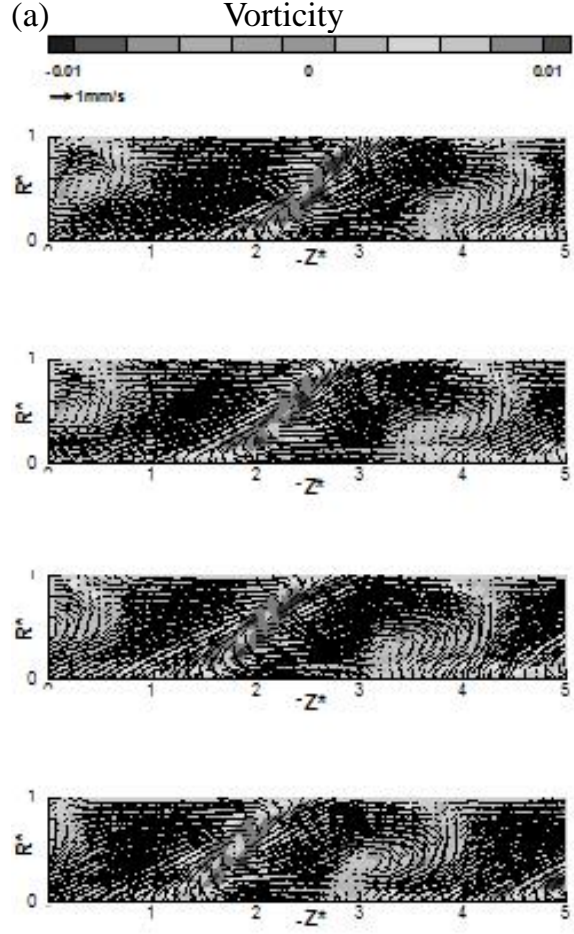

(b)

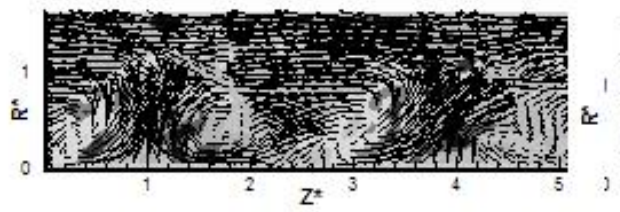

(c)
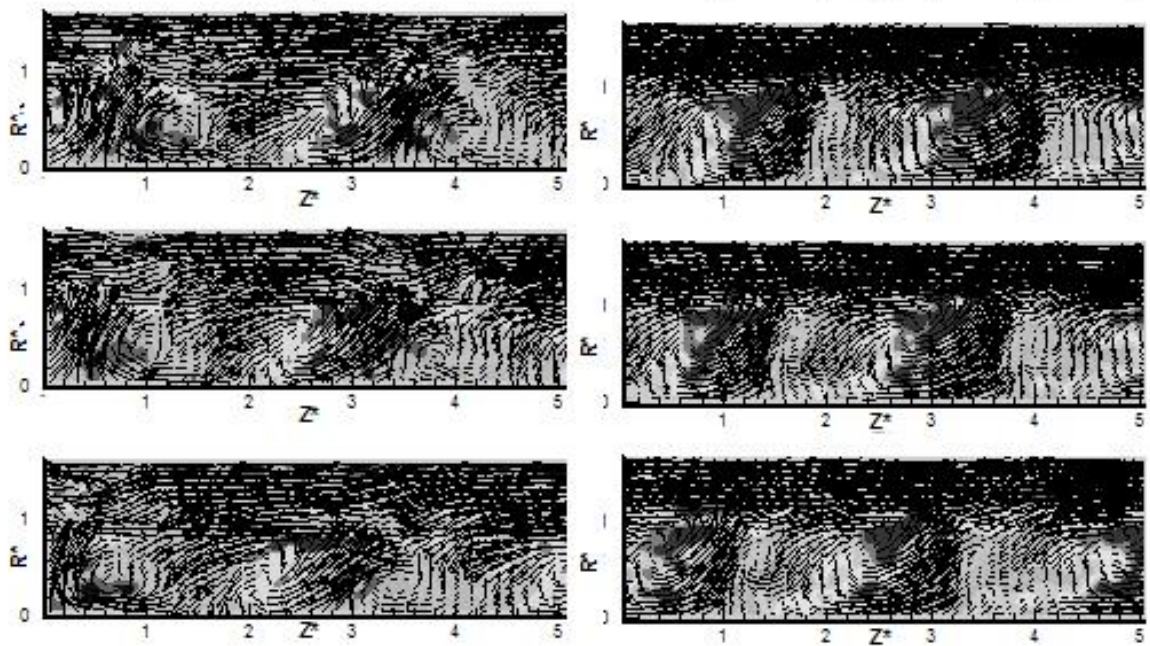
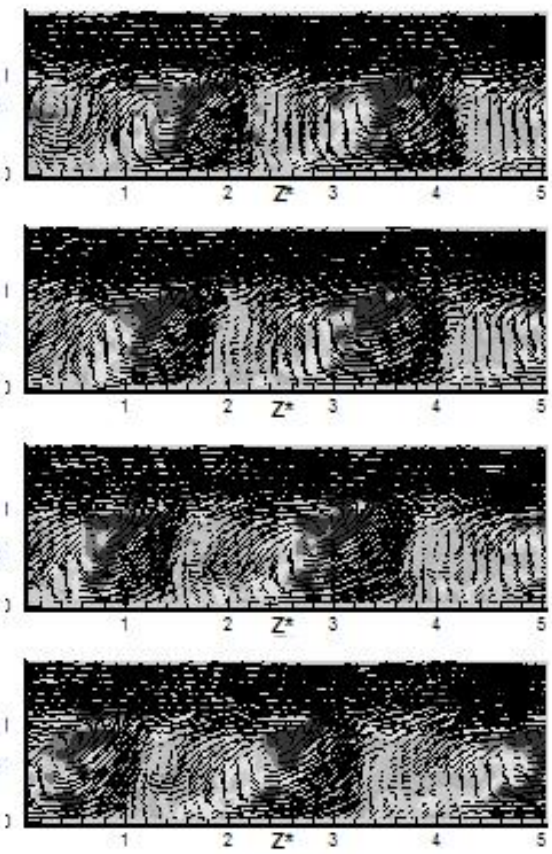

Fig. 3 Instantaneous velocity field of the helical vortex flow of at $\operatorname{Re}=96, \mathrm{Gr}=6700(\Delta t=0.4 \mathrm{~s})$

(a) The plain model (b) The 9-slit model (c) The 18-slit model,

\subsection{Helical Wavy Vortex Flow Regime}

In plain and 9-slit model, the transition from helical vortex flow to helical wavy vortex flow occurred, but for the 18-slit model, this flow regime did not appear. Fig. 4 shows the instantaneous flow fields of this flow regime when Re is 137 , the time interval between each velocity field is $1.2 \mathrm{~s}, 0.8 \mathrm{~s}$ and $1 \mathrm{~s}$ respectively. And also we can find small vortex in the slit space in 9-slit model, which means as the Re number increased, the vortex will appears in the slit space. And for plain model, this flow regime first occurred at $\mathrm{Re}=115$, but for the 9-slit model, which occurred at $\mathrm{Re}=107$. 
(a)
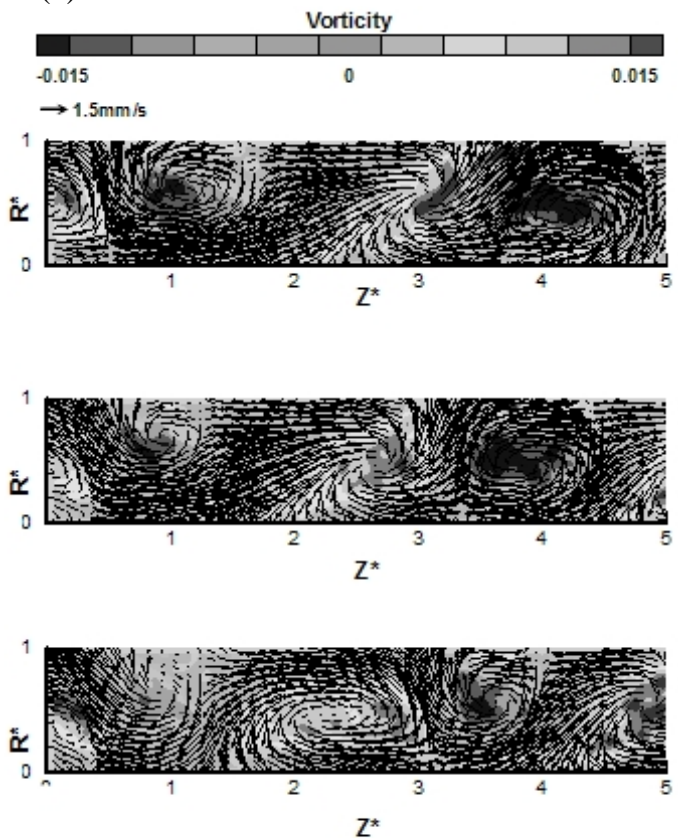

$Z^{*}$

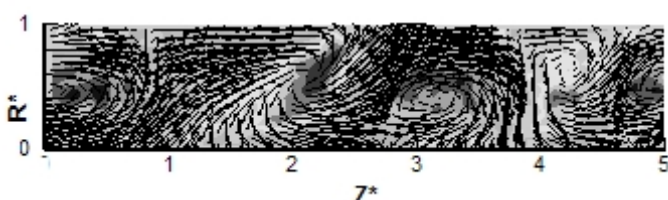

(b)

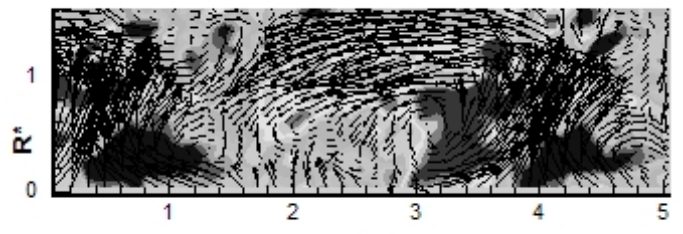

$\mathrm{Z}^{*}$
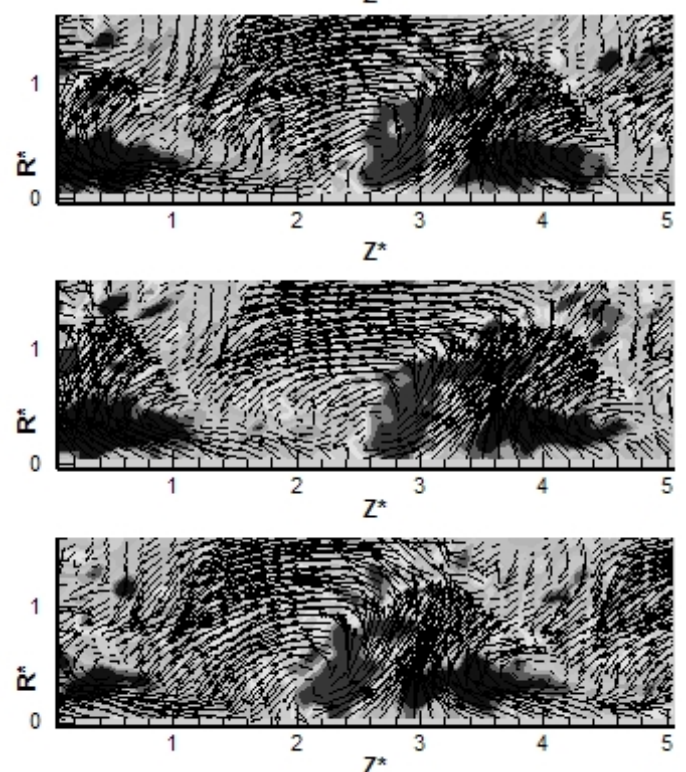

Fig. 4 Instantaneous velocity field of the helical vortex flow of at $\mathrm{Re}=137, \mathrm{Gr}=6700$

(a) The plain model (b) The 9-slit model

\section{Conclusion}

An experimental study was performed for a Taylor-Couette flow with temperature gradients between concentric cylinders. Through adjusting the rotation speed of the inner cylinder gradually, the effects of slit wall on the helical flow regimes were investigated. The helical vortex flow regime can be found in all the three models. The negative temperature gradients determine the direction of the rotation and movement of the helical vortex. However, the helical wavy vortex flow can only be found in the plane and 9-slit models. Comparing the results of plane and slits model, we can found the slit wall accelerated the transition to both helical flow regimes, and as the Re number increased, small vortex occurred in the 9-slit model.

\section{Acknowledgments}

This work was supported by Ph.D. Programs Foundation of Ministry of Education of China (20113227120012) and A Project Funded by the Priority Academic Program Development of Jiangsu Higher Education Institutions, and also supported by Priority Research Centers Program (2011-0031384) and Basic Science Research Program (2010-0024798) through the National Research Foundation (NRF) of the Republic of Korea funded by the Ministry of Education, Science and Technology.

\section{References}

[1] Taylor G. I., 1923, “Stability of a viscous liquid contained between two rotating cylinders,” Phil Trans R Soc Lond, 223:289343.

[2] Jones C. A., 1985, “The transition to wavy Taylor vortices,” J. Fluid Mech.157:135-62.

[3] Werely, S. T., Lueptow, R. M., 1998, “Spatio-temporal character of non-wavy and wavy Taylor-Couette flow,” J. Fluid Mech, 364:59-80.

[4] Rigopoulos, J., Sheridan, J., Thompson, M. C., 2003, “State selection in Taylor-vortex flow reached with an accelerated inner cylinder,” J Fluid Mech, 489:79-99.

[5] Marques, F., Lopez, J. M., 2006, “Onset of three-dimensional unsteady states in small-aspect-ratio Taylor-Couette flow,” J Fluid Mech, 561:255-277.

[6] Lee, S. H., Chung, H. T., Park, C. W., Kim, H. B., 2009, "Experimental investigation of slit wall effects on Taylor-Couette flow,” Fluid Dynamics Research. 41:1-12.

[7] Lepiller, V., Goharzadeh, A., Prigent, A., Mutabazi, I., 2008, "Weak temperature gradient effect on the stability of the circular Couette flow,” The European Physical Journal B, 61:445-455.

[8] Hayase T., J. Humphrey A. C., Greif R. 1992, "Numerical calculation of convective heat transfer between rotating coaxial cylinders with periodically embedded cavities,” J. Heat Trans. 114:589-597.

[9] Lee, Y. N., Minkowycz, W. J., 1989, "Heat transfer characteristics of the annulus of two-coaxial cylinders with one cylinder 
rotating,” Int. J. Heat Mass Transfer, 32:711-722.

[10] Cole J. A., 1976, “Taylor-vortex instability and annulus-length effects,” J. Fluid Mech. 75:1-15.

[11] Liu D., Lee S. H., Kim H. B., 2010, "Effect of a Constant Radial Temperature Gradient on a Taylor-Couette Flow with Axial Wall Slits,” Fluid Dyn. Res., 42, doi:10.1088/0169-5983/42/6/065501. 